\title{
Virtualidades e realidades latino-americanas: a web e o ensino de História para além das fronteiras nacionais
}

\section{Potentialities and realities in Latin America: the web and the history teaching across national borders}

Júnia Sales Pereira*

Sonia Regina Miranda ${ }^{* *}$

Quando pensamos, nos dias de hoje, no potencial educativo e comunicativo disparado pelas tecnologias da informação, com ênfase para a internet, tendemos a refletir sobre o que esse arsenal de informação nos possibilita em termos de acesso a novos mundos. Navegamos por ambientes que potencializam a aquisição não só de documentos como também de textos e imagens favoráveis ao estudo da História e ao seu tratamento didático em sala de aula.

Entretanto, também é comum pensarmos sobre como a internet hoje vem se convertendo em uma espécie de novo espaço público na contemporaneidade. Nesse espaço, não podemos perder de vista as novas contradições engendradas por aquilo que Nestor Canclini nos adverte acerca das novas fantasmagorias geradas pelas dinâmicas envolvidas no binômio "ser espectador internauta" versus "ser visitante num espaço que possui autorias, intencionalidades e propriedades". Esse desafio nos impõe uma importante tarefa no sentido de educarmos os estudantes para a operação com o procedimento histórico, fornecendo-lhe ferramentas que possam funcionar como filtros de leitura para a montanha de informações que nos são apresentadas pela web.

Indo um pouco mais além, a internet nos possibilita, ainda, refletirmos acerca de novos modos de operação com as práticas de Memória, na medida em que talvez nunca se tenha produzido, armazenado e divulgado tantos suportes de memória, especialmente nas redes sociais.

\footnotetext{
* Doutora em História, Universidade Federal de Minas Gerais (UFMG); Ministério da Educação. juniasales@gmail.com

** Doutora em Educação, professora da Universidade Federal de Juiz de Fora (UFJF). sonia. miranda@ufjf.edu.br
} 
Mas, se pensarmos na ampliação desse leque de possibilidades, veremos que a internet também se abre enquanto canal disparador de diálogos entre sujeitos que podem se encontrar através de novos instrumentos mediadores. Nesse sentido, essa tecnologia projeta-se como uma rede aberta à conexão de pesquisadores, professores e estudantes, de diversas regiões brasileiras, diversas cidades e diversos países. Possibilita, portanto, a construção de novos circuitos de aprendizagem e novos modos de operar com o encontro de sujeitos em suas identidades e alteridades.

Foi nessa direção que pensamos, para este Dossiê, colocar em movimento vozes de pesquisadores latino-americanos que vêm se dedicando a investigar o campo do ensino de História. Numa entrevista virtual durante o mês de setembro de 2014, foi possível reunir - no que agora se converte em um texto único que nos permite estabelecer olhares em torno de problemáticas latino-americanas - quatro pesquisadores selecionados:

Sebastian Plá é docente e pesquisador da Universidad Pedagógica Nacional do México, onde realizou seu Doutorado em Pedagogia. É licenciado em História pela Universidad Iberoamericana. Dedica-se, em suas pesquisas, à temática do Ensino de História. É Membro do Sistema Nacional de Investigadores (nivel I) e do Consejo Mexicano de Investigacion Educativa (COMIE).

Lucía Valencia Castañeda é docente e pesquisadora na Universidad de Santiago, no Chile, onde atua no campo da Didática e da formação de professores de História. Doutorou-se em Didáctica de las Ciências Sociales pela Universitat Autònoma de Barcelona.

Miguel Angel Jara é docente e investigador na área de didática das Ciências Sociais e História nas Faculdades de Ciências da Educação e Humanidades da Universidad Nacional del Comahue, no norte da Patagônia, na Argentina. É doutor em Didáctica de las Ciencias Sociales pela Universitat Autònoma de Barcelona.

Martha Cecília Gutierrez Giraldo é professora e investigadora na Universidad Tecnológica de Pereira, na Colômbia. É graduada em Psicologia e vem se dedicando a investigar as questões relativas ao ensino de História e Ciências Sociais, com ênfase na reflexão sobre 
a formação de professores e cognição humana. É Doutora em Ciências Pedagógicas pela Universidad de La Habana e Pós-Doutora pela Universitat de Barcelona.

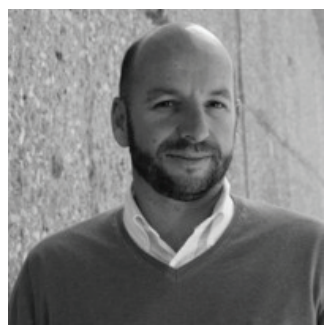

Sebastian Plá

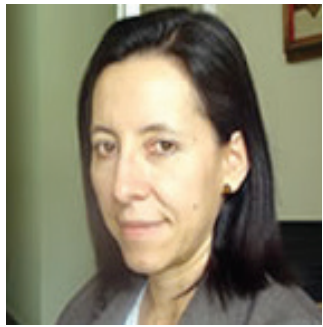

Martha Cecília Gutierrez

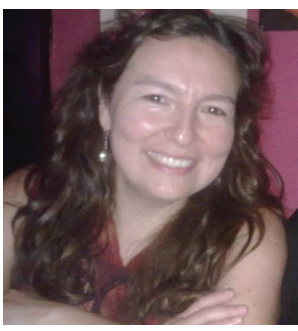

Lucía Valencia Castañeda

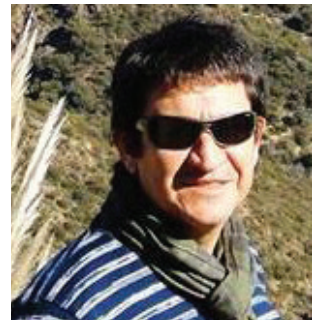

Miguel Angel Jara

Sonia e Júnia: Como tem sido sua experiência de investigar o ensino de História em seu país? Que dificuldades você encontra? Quais são os problemas mais recorrentes? $Q u e$ vantagens você observa diante desse investimento profissional e acadêmico?

Sebastian Plá: La investigación en enseñanza de la historia en México ha tenido un crecimiento exponencial en el último lustro. Sobre todo a partir de los encuentros de la Red de Especialistas en Docencia, Difusión e Investigación en Enseñanza de la Historia (reddieh). En el encuentro de septiembre de 2014 se recibieron más de 180 ponencias. También han aumentado, muy poco, las publicaciones en revistas indexadas, siendo tres o cuatro autores quienes lo han hecho. Casi todas en revistas de educación y solo dos en revistas de historia. Los investigadores, por lo general, no pertenecen al sistema nacional de investigadores. 
Asimismo, han crecido los estudios de posgrado, con cuatro maestrías. En el doctorado de pedagogía de la UNAM tengo noticias de una tesis concluida y tres en proceso. Sin embargo, teórica y metodológicamente existen grandes rezagos. El principal quizás sea el desconocimiento de autores relevantes del mundo anglosajón, francés, italiano y latinoamericano. Ni se diga Turquía, el Magreb o Australia. Hay, a causa de la lengua, un predominio casi absoluto de autores españoles.

El otro gran problema es la incapacidad de muchos investigadores o estudiantes de posgrado de ver más allá de la historiografía de los Annales en su segunda generación y de un mirada cognitiva de los procesos de aprendizaje (conductismo oculto tras constructivismo). Por ejemplo están fuera de la discusión la cultura escolar, los programas de estudio dentro de contextos políticos curriculares más amplios, los usos públicos de la historia, la enseñanza de la historia como violencia epistémica, etc.

En resumen, el interés en la investigación en enseñanza de la historia ha aumentado y se ha conjuntado una red relativamente sólida (aunque muy localista o ibérico referencial). El problema es que es muy poca la investigación que tenga nivel para publicarse en revistas de educación o de historia nacionales o internacionales de alto impacto. Sigue siendo una investigación "parroquial".

Lucía Valencia: La investigación en la enseñanza de la Historia y las Ciencias Sociales en Chile, es un área de desarrollo reciente. Comienza a implementarse sólo a fines de la década de 1990, coincidente con los cambios políticos iniciados en el país con unos años de antelación, y la puesta en marcha de un nuevo Marco Curricular, que abandonó la opción enciclopedista de la enseñanza, por una que comienza a poner énfasis en el desarrollo de habilidades para la comprensión del medio social. Dicha reforma y el Ajuste Curricular del 2009, dieron una nueva orientación al currículum, caracterizado por propósitos que ponen énfasis en la construcción de una sociedad democrática y el respeto de los derechos humanos; enfoques conceptuales que relevan la historia reciente y categorías como la memoria histórica y la ciudadanía; y la preeminencia dada a las habilidades para el desarrollo del pensamiento histórico y social Estos cambios dieron lugar a un prolongado debate sobre las finalidades de la enseñanza y cómo estas se resolvían en el ejercicio práctico de las salas de clases. 
Desde entonces, han tenido lugar investigaciones en la enseñanza de la Historia y las Ciencias Sociales a través de tesis de doctorado realizadas en el país y en el extranjero, especialmente en España y en Catalunya, y proyectos financiados por el Estado.

En 1996 se crea la Asociación Chilena de Didáctica de la Historia, la Geografía y las Ciencias Sociales, que en la década del 2000 organiza Jornadas Nacionales de Didáctica en diversas universidades del país, y la primera de carácter internacional en la ciudad de Osorno en 2006, inaugurando las incipientes oportunidades de difundir y debatir en cuestiones como las definiciones epistemológicas de este campo de investigación que comenzaba a abrirse camino, además de constatar las profundas necesidades de desarrollo que requería el área.

El año 2016 Chile será sede del tercer encuentro de la Red Iberoamericana de Didáctica de las Ciencias Sociales, fundada en Colombia el 2012. Y el 2015 deberían inaugurarse los primeros programas de post grado en Didáctica de la Historia y las Ciencias Sociales impartidos en nuestro país.

Una de las principales desventajas se relaciona con las dificultades, muy comunes en este campo de investigación, de acceder a los espacios de las aulas escolares, por definición, el escenario natural de la investigación sobre el aprendizaje y la enseñanza de la Historia. Las resistencias provienen fundamentalmente de los propios docentes, poco acostumbrados a que la presencia de un observador en su sala de clases tenga finalidades investigativas, la que relacionan, en cambio, con objetivos de evaluación de su desempeño.

La corta historia de este campo de investigación ofrece la dificultad de no contar con una masa crítica muy consolidada en este plano, pero paradojalmente constituye también una gran ventaja. Se trata de un campo donde hay mucho por hacer y que en los últimos años se ha visto beneficiado por un crecimiento explosivo de recursos destinados a formar especialistas de post grado en esta área del saber y a la realización de investigaciones encaminadas al desarrollo del campo específico de la didáctica disciplinar, especialmente en relación con la calidad de la formación inicial de profesores. Desde mi punto de vista, este nuevo escenario ofrece una expectativa muy auspiciosa para el desarrollo de la investigación en la enseñanza de la Historia y las Ciencias Sociales. 
Martha Cecília: Romper con la tradición de lo que ha sido la enseñanza de la historia es difícil en una sociedad como la colombiana, donde 60 años de conflicto interno, nos han polarizado demasiado y han invisibilizado a muchos grupos sociales.

Sin embargo y no obstante el panorama anterior, investigar en la enseñanza de la historia y de las ciencias sociales en general, es una oportunidad para construir otras visiones de la Didáctica de la Historia y de las Ciencias Sociales, que nos permita compartir con los estudiantes formas más críticas y participativas de aprender la historia, construir ciudadanía y democracia.

Miguel Ángel: Mi experiencia en la docencia y en la investigación en el ámbito universitario es relativamente reciente, de los 12 años de trabajo en la universidad, los últimos 3 lo he dedicado exclusivamente a este ámbito de la formación. Hasta entonces y por más de 18 años he desarrollado mis actividades profesionales, simultáneamente, en diferentes niveles del sistema educativo. Lo que sin dudas ha contribuido en una visión amplia sobre los problemas vinculados a la enseñanza y el aprendizaje de la historia y de las ciencias sociales.

Dicho esto y circunscribiéndome a mi experiencia en la investigación recupero las posibilidades que me ha ofrecido indagar por una década, junto a un equipo de profesores y profesoras universitarios, las complejas relaciones y tramas entre historia-memoria y enseñanza focalizando en los procesos de la historia reciente/presente (HRP) de nuestro país y el entorno.

Digo posibilidades porque las investigaciones, de corte cualitativo y crítico, que hemos desarrollado nos ha permitido conocer las perspectivas teóricas y prácticas que orientan las acciones pedagógicas que el profesorado de la región lleva a cabo en las clases de historia y de ciencias sociales en las escuelas públicas de nivel primario y medio. También los modos, y las relaciones que las y los jóvenes realizan sobre estos procesos candentes e indeterminados, no cerrados y de plena actualidad.

La investigación en la enseñanza y el aprendizaje de la HRP se ha convertido en una línea que diversos equipos llevan adelantes en sus universidades (Rosario, Córdoba, Salta, Buenos Aires, Tucumán, entre otras) y también en otras universidades del continente. Evidentemente la problemática ha generado interés, en la medida que es una línea de investigación que procura conocer, 
para interpretar, las dificultades que se evidencian en la enseñanza y aprendizaje del conocimiento de lo social y para ofrecer alternativas para su mejora.

Si bien los resultados de las investigaciones son diversos y para nada pretenden generalizar, son indicios que nos permiten sostener que los problemas son cada vez más comunes y globales, tal como lo sostiene Joan Pagés. Sin embargo, a modo de ejemplo podríamos destacar que una de las dificultades profundas y recurrentes se encuentra en la Formación Inicial del Profesorado, con algunos matices y especificidades según de qué institución formadora se trate.

En términos metodológicos, por suerte, podríamos decir que se ha abierto un camino interesante. Los procesos metodológicos, construidos por una comunidad de investigadores/as en didáctica de la historia, se van consolidando como estrategias investigativas que permiten mirar-se y mirar-nos en los escenarios diversos en los cuales se desarrolla la enseñanza y la investigación. Las técnicas construidas en la investigación cualitativa (entrevistas en profundidad, cuestionarios, relatos de vida, narrativas escolares, estudios de caso, entre otras), en nuestras investigaciones, son instrumentos potentes que nos han permitido adentrarnos e involucrarnos en los casos construidos. Sumado a ello un trabajo colaborativo y participante en el proceso investigativo. En este sentido festejo las innovaciones, aunque debemos admitir que, por ahora, no se ha constituido en una práctica generalizada en los equipos de investigadores/as.

En el marco de las dificultades podríamos identificar las relacionadas a las presupuestarias, que no es menor, en la medida que no permite incorporar nuevas generaciones en la investigación en el campo de la didáctica de la historia, también los criterios de evaluación de los proyectos de investigación que no siempre contemplan los aportes de la investigación educativa. Por otra parte no es una preocupación ministerial promover y sostener investigaciones de este tipo, evidentemente no son consideradas relevantes para la mejora en la educación de la ciudadanía. Paradoja de un problema que denuncia y anuncia más posibilidades que límites.

Pienso que esta dificultad, entre otras, son las que deberían considerarse como fundamentales para que, a partir de la investigación, se promuevan cambios en la enseñanza. Ocuparse de los problemas, sistematizarlos y evidenciarlos es un camino viable que abre un universo de posibilidades. Y este, sostengo, es el insumo de nuestro campo tan fértil como apasionante. 
Sonia e Júnia: Como se organizam, em grandes linhas, os cursos de formação de professores de História em seu país? Como você avalia o cenário contemporâneo no tocante a essa relação?

Sebastian Plá: La formación de profesores en México (pueden ver mi publicación sobre el tema en www.academia.edu/2644376/Un_panorama_sobre_la_formacion_de_docentes_en_historia_en_Mexico_An_overview_on_ the_training_of_history_teachers_in_Mexico) se divide según el nivel educativo del que hablemos. Para educación preescolar y primaria (3-12 años) no hay maestros de historia. Son formados en la escuela normal con graves deficiencias en casi todas las asignaturas. En algunos casos, los docentes cursan talleres de actualización desarticulados y raramente dedicados a la historia. Predomina matemáticas y español. En secundaria los maestros tienes dos procedencias: docentes formados en la normal superior con especialidad en historia y docentes provenientes de las universidades, pero de carreras muy disímiles: geógrafos, abogados, historiadores, literatos, antropólogos, economistas. A modos de ejemplo inverso. Dirijo una tesis de maestría de enseñanza de la historia y niños ciegos. Mi alumna es historiadora y maestra de historia, pero también es docente de educación física, aunque no sabe nada del tema pero necesita el dinero. Lo mismo pasa en sentido contrario. Es un sistema en el que Neymar Jr. imparte cátedra en la USP y Ciro Cardoso mete gol en el Maracaná. En educación media superior (15-18 años) sucede casi lo mismo que en secundaria, aunque un poco más controlado.

Sin embargo, creo que poco a poco la enseñanza de la historia, por lo menos en las grandes ciudades y en las instituciones de educación media superior y a veces secundarias, privadas y públicas con mejor rendimiento académico, ha mejorado mucho. Ha reducido un memorismo insensato y el nacionalismo revolucionario se ha matizado, aunque no desaparecido. El problema de la enseñanza de la historia es más amplio y está enmarcado en la inequidad y exclusión educativa de todo el sistema. Los problemas más graves están en los sectores marginados, donde no se capacita a los docentes o son profesores sin experiencia, mientras que en los barrios con mayor poder adquisitivo, el problema técnico, más no el político, está relativamente resuelto.

Lucía Valencia: En coherencia con la respuesta a la pregunta anterior, la formación de profesores de Historia en Chile está teniendo importantes modificaciones. La tendencia general es relevar la formación didáctica y 
pedagógica respecto de la formación disciplinaria, tradición de larga raigambre en la formación del profesorado en nuestro país.

La formación didáctica adquiere cada vez más peso en las estructuras curriculares de la formación del profesorado de Historia. Lo mismo ocurre con la importancia dada a las experiencias prácticas en situaciones reales de aula. La tendencia es que éstas se adelanten en el proceso formativo de los futuros profesores y que se vinculen con experiencias de reflexión en los cursos de didáctica.

La situación anterior está siendo reforzada por políticas estatales, que están propiciando mediante el financiamiento de proyectos de mejoramiento de la calidad de la formación inicial docente, la contratación de profesores de didáctica de las especialidades en los Departamentos disciplinarios.

Estos cambios son coherentes con las tendencias más contemporáneas e innovadoras en el campo de la formación del profesorado.

Martha Cecília: La enseñanza de la historia en las facultades de educación y los programas de formación de docentes, siguen estando muy centrados en la disciplina, es decir formar historiadores e investigadores de la historia, pero se desconoce o se descuida la formación en la didáctica de la historia.

Miguel Ángel: Argentina posee una geografía diversa a lo largo y ancho del territorio. En este sentido podríamos afirmar que también son diversas las experiencias formativa de profesores y profesoras en historia y ciencias sociales. La formación de profesores/as en la disciplina no es privativa de la Universidad. También los Institutos de Formación Docente (IFD), no universitarios, poseen en su haber una trayectoria importante en la formación de profesores/as para la educación media. A partir de la creación del Instituto Nacional Formación Docente (INFD), en el marco de la Ley de Educación Nacional (LEN) del año 2006, los IFD se embarcaron en un proceso de reformas de sus planes de estudios sobre la base de algunos lineamientos generales establecidos por el INFD para toda la geografía nacional. Si bien cada jurisdicción (Provincia) construyó de modo diverso su nuevo currículo, la formación se extendió de tres a cuatro años, con una fuerte vinculación con las instituciones de nivel medio o primario con una importante formación pedagógica y didáctica especifica.

Este proceso no se condice con la Formación Universitaria del Profesorado. Si bien las experiencias no son generalizables, son pocas las 
Universidades Nacionales que han revisado sus planes de estudios y promueven una formación didáctica de los/as futuros/as profesores/as.

La Facultad de Humanidades de la Universidad Nacional del Comahue (UNCo), por más de cuatro décadas, forma a profesores y profesoras en historia. El actual plan de estudio vigente desde 1985 con algunas reformas, está organizado en 4 áreas de conocimientos disciplinares e historiográficos, dependientes del Departamento de Historia de la Facultad de Humanidades. De las 29 asignaturas del plan - que reconoce un ciclo de formación común y un ciclo de formación específica para cada una de las titulaciones - sólo 4 corresponden a las asignaturas llamadas “pedagógicas”, que son Psicología General y Evolutiva, Didáctica General y Especial, Problemas Fundamentales de la Educación y Prácticas Docentes y están ubicadas en el quinto año del Profesorado. Todas dependientes de la Facultad de Ciencias de la Educación, 2 de ellas - en la que me desempeño como profesor formador - dependen del Departamento de Didáctica, el área de Didáctica de las Ciencias Sociales: Orientación historia.

Frente a esta estructura curricular, y en función de lo establecido en el plan de estudios, los y las estudiantes que han cursado y aprobado las asignaturas correspondientes al Ciclo Introductorio y al Ciclo Profesional (cuyos objetivos se centran en la profundización de los contenidos específicos de la carrera, en el debate de las corrientes historiográficas, en el aprendizaje de métodos y técnicas y en fomentar el espíritu crítico y el sentido histórico) estarían en condiciones para la realización y puesta en ejercicio de sus Prácticas Docentes, en una institución educativa de nivel medio o superior. Evidentemente subyace en esta concepción una idea aplicacionista de la didáctica según puede verse en el plan de estudios.

En general las propuestas formativas, desde una perspectiva didáctica y pedagógica del profesorado, varían a lo largo y ancho de la geografía nacional. Aunque hay un punto en común: finalizada la formación disciplinar y la escasa formación para la enseñanza, los/as estudiantes realizan su prácticas docentes, en un tiempo escaso material y curricularmente. Sin mayores relaciones y vínculos con la escuela secundaria, lugar donde se insertarán como profesionales.

En este sentido, considero oportuno destacar que la formación universitarita del profesorado va por caminos distintos a los que se producen en la 
escuela secundaria. Al menos en la región, (Provincias de Rio Negro y Neuquén) en las se han producido reformas y contrarreformas curriculares y educativas, con ciertas innovaciones, que no han sido contempladas por las instituciones formadoras. La ausencia de diálogos entre niveles del sistema formador, es un obstáculo importante si pensamos en la posibilidad de innovación en la enseñanza de la historia y de las ciencias sociales. ¿Para qué contextos de inserción profesional se forma al profesorado? Este es el dilema a repensar y el compromiso por asumir.

Hace un tiempo que vengo sosteniendo que es necesario de rescatar el carácter inacabado del conocimiento y de la formación. Enseñar a enseñar, reflexionando sobre qué significa aprender historia y qué significa aprender la historia para enseñarla, contribuirá a la construcción de futuro, descartando una única realidad viable, observando la existencia de diversos horizontes posibles.

Sonia e Júnia: Como se organizam os currículos de ensino de História em seu país no ensino fundamental e médio? Existem programas prescritos rigidamente pelos governos? Ou há autonomia de gestão por parte dos docentes?

Sebastian Plá: El sistema educativo obligatorio en México tiene, desde 2011, cuatro niveles: preescolar (3-6 años), primaria (6-12), secundaria (12-15), media superior (15-18). Cada nivel tiene subdivisiones, por lo que aquí les expongo la general, donde cursan la mayoría de los estudiantes.

Las tres primeras llevan solo hay un currículum nacional y obligatorio. Historia como asignatura se imparte en primaria según el grado (puede verse el cuadro); tercero: historia de la entidad federativa; cuarto: historia de México antiguo y colonial; quinto: historia de México XIX y XX; sexto: historia antigua hasta los viajes de descubrimiento portugueses y españoles.

El plan está pensado como un continuo, como educación básica, por lo que se retoma en segundo de secundaria historia universal de 1500 a la actualidad. En Tercero vuelven a ver toda la historia de México. Independientemente del nivel se deben desarrollar las competencias de tiempo y espacio histórico, análisis de fuentes y conciencia histórica.

En EMS la diversidad es tal que nunca acabaría de explicarlo. Un colega escribió un libro de cuatrocientas páginas tratando de desenmarañarlo. Pero desde 2008 hay competencias que todos deben desarrollar, tanto genéricas 


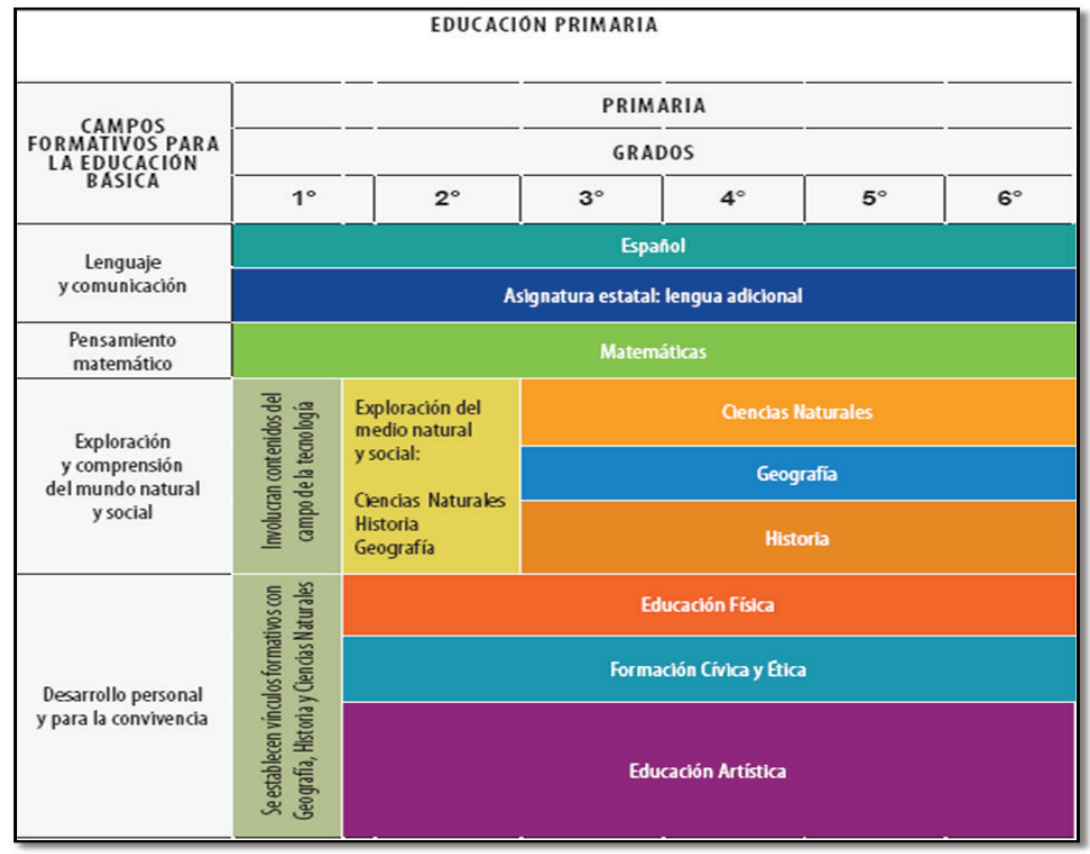

como disciplinares. En estas últimas se incluyen a las ciencias sociales y a su vez a la historia. Hablan de ciudadanos críticos y reflexivos que identifican el conocimiento como construcción constante, sitúa hechos históricos fundamentales, interpreta la realidad social a partir de procesos históricos locales, valora la diversidad, relaciona política, economía, cultura y geografía parte pensar la realidad, es emprendedor, competitivo y democrático.

El sistema educativo mexicano (SEM) es muy jerárquico y autoritario. No hay libertad docente alguna. A modo de ejemplo: el programa dice que el maestro debe ser autónomo y para esto se creó un instituto de evaluación (INEE) que seguirá puntualmente al docente para medir que tan autónomo y creativo es de acuerdo al programa prescrito. Y si no fue lo autónomo que se le pidió, le bajan el salario. El maestro de preescolar, primaria y los de historia de secundaria están en esta circunstancia.

Lucía Valencia: Existe un Marco Curricular obligatorio para la enseñanza primaria y secundaria. Está compuesto de Contenidos Mínimos Obligatorios y Objetivos Fundamentales. Los colegios tienen libertad para diseñar 
Programas de Estudio (Aprendizajes Esperados, Secuencias Didácticas, Actividades y Evaluaciones) a partir del Marco Curricular prescrito.

En la práctica son muy pocos los colegios que diseñan sus propios Programas y en general trabajan con las propuestas emanadas desde el Ministerio de Educación, el que además ha desarrollado una importante política de licitación de textos escolares que son distribuidos gratuitamente en las escuelas.

A la influencia anterior ejercida por el Estado, se suma desde mi punto de vista la más importante, la realización de una prueba estandarizada de carácter obligatorio que se aplica entre 4 y 5 oportunidades en una trayectoria escolar de 12 años de duración.

La extensión del currículum y la presión permanente de esta evaluación, son señaladas por los profesores como factores que los obligan y les impiden implementar el currículum con libertad y flexibilidad.

Martha Cecília: El gobierno colombiano integró la enseñanza de la historia en las ciencias sociales y promulgó unos lineamientos curriculares y unos estándares de competencias en ciencias sociales, que rigen a nivel nacional.

Los lineamientos curriculares y los estándares de competencias en ciencias sociales son la base para la construcción de los currículos, los planes de estudio y del área en cada institución educativa, que a su vez, son el eje para la actividad académica de los docentes en las aulas de clase.

Miguel Ángel: La verdad, hasta donde conozco, son bastantes heterogéneos. Para el nivel primario encontramos más experiencias de construcciones curriculares sostenidas en el tiempo. No sucede lo mismo con el nivel secundario. En argentina, al menos desde el proceso democrático abierto en 1983, hemos transitado por dos leyes nacionales de educación y las construcciones curriculares en concordancia con los principios que las leyes promovían. La primera, La Ley Federal de Educación (LFE 24195/93), fue muy resistida por el colectivo docente y, en algunos casos, por la comunidad toda. Resistencia generada no solo por haber sido poco participativa su elaboración, por la perspectiva de la tecnología educativa en la que se sostenían sino, fundamentalmente, por el corrimiento del estado en materia educativa. Lo que implicó un avance de las políticas neoliberales aplicadas al sistema educativo, en sus diferentes niveles. 
La LFE, de la década de los 90, se materializó en diversos diseños curriculares, en todos los niveles de la educación obligatoria, de manera diversa y no en todas las provincias del territorio nacional e incluso significó, para algunas jurisdicciones, una contrarreforma a las experiencias innovadoras y exitosas que habían iniciado.

En este sentido se diseñó una estructura del sistema educativo nacional que pretendió establecer en cada nivel criterios sostenidos en un esquema que pretendía: a) la elaboración y acuerdos sobre los contenidos básicos comunes (CBC), instancia Nacional; b) contextualización de los CBC, en función de las realidades regionales, estableciendo criterios de orientación general y especifica, en el marco jurisdiccional; y c) la formulación de Proyectos Curriculares a nivel Institucional y áulico, en consonancia con los criterios establecidos en los niveles anteriores. Modalidad que no logro materializarse en la amplia participación que presuponía.

El cambio en las políticas educativas, que dio como resultado un "nuevo sistema educativo” de carácter federal, reorganizó el sistema formador en 13 años de escolaridad. Los 10 primeros años eran obligatorios (de 5 a 14 años de edad) organizados en tres ciclos, lo que constituía la Educación General Básica (EGB) y un años para el nivel inicial. Los tres restantes, pos obligatorios, correspondieron al Polimodal cuyas orientaciones se centraron en el trayecto técnico profesional y pre universitario.

En el año 2006, una nueva Ley Nacional de Educación (LEN) es sancionada y modifica nuevamente la estructura del sistema educativo nacional. Amplia la obligatoriedad de 3 a 17 años, bajo una organización tradicional de la escolaridad: Nivel inicial (3 a 5 años), nivel primario (6 a 12 años) y nivel secundario o medio (13 a 17 años).

En cuanto a la construcción curricular, si es prescriptiva o no, podríamos decir que durante la LFE no se pudo avanzar (por la resistencia de la que hablábamos), en un currículo de alcance nacional. Cuestión que si se sigue construyendo en el marco de la LEN. Para el primer caso, las editoriales fueron las que introducen un currículo a partir de los libros te textos escolares. Para el segundo caso, fue el Ministerio de Educación de la Nación, quien distribuyó textos para estudiantes y para profesores. Los NAP (Núcleos de Aprendizaje Prioritarios) son los que hoy encontramos como orientadores de las prácticas educativas, sumado a diversidad de producciones y programas didácticos, con 
una clara postura política que orienta el diseño y se desprende del espíritu de la LEN.

Sin embargo, dado este proceso, existe diversidad de experiencias tanto individuales, colectivas o institucionales, que generan prácticas alternativas y de muy buenas construcciones pedagógicas-didácticas. Lamentablemente son pocos los casos. Esto da cuenta que el cambio del currículo, por si mismo, no garantiza el cambio en la práctica, si no va acompañado de un programa de formación continuada y sostenida en el tiempo.

Sonia e Júnia: Que demandas são mais recorrentes por parte dos professores de História em seu país?

Sebastian Plá: Fuera de los sindicatos y las condiciones salariales y materiales de la escuelas, sobre todo en regiones marginadas del sur del país, las demandas políticas son muy pocos. Parte fundamental del autoritarismo del SEM se basa en el sindicato.

En los cursos de actualización tienden a pedir lo mismo que en muchos otros lugares: estrategias que le resuelvan cómo enseñar. Cuando trabajo con ellos en cursos de 20 horas, dedico por lo menos cinco a catarsis, en los que expresan su malestar por las condiciones laborales y piden estrategias. Luego imparto mi mirada cultural y política. En un curso de autoevaluación sobre necesidades de actualización que hice con una estudiante de posgrado y que estaba dirigido a profesores de educación media, básicamente solicitaron al final de taller cursos sobre: culturas juveniles, nuevas historiografías y, no pudimos evitarlo, didáctica entendida como meras estrategias.

Lucía Valencia: Las demandas de los tres últimos años se insertan en un proceso general de la sociedad chilena que exige mejorar la calidad de la educación. Las exigencias son por terminar con las prácticas que permiten hacer de la educación un negocio lucrativo y que el Estado tenga más injerencia en el financiamiento de este derecho social.

Los profesores demandan mejorar sus condiciones laborales, fundamentalmente que disminuyan las horas efectivas de trabajo en la sala de clases a favor de tiempo remunerado destinado a la preparación de la enseñanza.

En los últimos cuatro años los profesores de Historia se movilizaron por dos demandas curriculares específicas. Su oposición a la disminución de las horas de Historia y Ciencias Sociales en el currículo escolar, en favor de 
aumentar las horas de lenguaje y matemáticas. En segundo lugar, su oposición a que los años del gobierno de Augusto Pinochet fueran denominados régimen militar en lugar de dictadura. En ambos casos las protestas de los profesores resultaron exitosas en sus demandas.

Otra demanda que cada vez tiene más adeptos, es que se ponga fin al SIMCE, el sistema de evaluación estandarizado que se aplica en Chile cada vez con más frecuencia durante la trayectoria escolar. Su objetivo es medir la calidad de la enseñanza, pero sus consecuencias han sido muy nocivas. Entre las que se encuentra la estigmatización de las escuelas con malos resultados, siempre pertenecientes a sectores más postergados socialmente. Los profesores reclaman la descontextualización de esta evaluación, la gran cantidad de recursos que significa, los que podrían destinarse a mejoras más directas en las condiciones de la enseñanza.

Martha Cecília: En el país hay visiones hegemónicas de la historia, que invisibilizan a las mujeres, los negros, los indígenas, los pobres, entre otros grupos poblacionales denominados minorías étnicas.

Los currículos y los libros de texto que circulan en el país muestran una versión de la historia muy parcializada, la formación política y ciudadana de los estudiantes cada vez es más escasa, entre muchas razones, porque el tiempo dedicado a toda el área de las ciencias sociales es cada vez más corto, dos o tres horas semanales de clases teóricas para responder a las competencias que exige el Ministerio de Educación, en detrimento de la formación del pensamiento social y de la formación ciudadana y democrática que requiere este país, para contribuir a la solución de los conflictos sociales de de vieja data.

Miguel Ángel: Las demandas son diversas. Una de las principales y que no es menos, al menos desde mi punto de vista, son las condiciones laborales en las cuales se lleva a cabo la práctica docente y de enseñanza. Es histórico el reclamo de condiciones edilicias, de salarios dignos, de aulas superpobladas, de recursos y materiales, de asistencia profesional para los centros educativos, de seguridad, entre otros tantos.

En relación a la formación continuada, la demanda también es importante. No es que no se ofrezcan, desde diversidad de instituciones incluida el estado, sino que son esporádicas, no son en servicio y muchas veces onerosas para los salarios docentes. 
Desde mi experiencia en diversidad de cursos de capacitación, junto a la del equipo que integro, da cuenta de una necesidad de actualización profesional que pueda dar cuenta de los problemas de aprendizajes en los actuales contextos. Posibilidades de repensar la enseñanza de las disciplinas escolares con nuevos enfoques, materiales y perspectivas que la formación inicial no ha brindado.

Siento que el sistema "aplasta" y provoca "desgano" en el profesorado. La discontinuidad en las clases, la ausencia de proyectos políticos educativos claros y sostenidos en el tiempo, más allá de los gobiernos de turno, son los aires que se respiran en las instituciones educativas. Lamentablemente, y frente a un mundo de incertidumbres, no ofrecemos políticas claras que permitan construir nuevas certezas que recuperen el sentido profundo de la educación para la autonomía, el conocimiento y la toma de decisiones de las futuras generaciones.

Sonia e Júnia: Como você avalia sua condição de ser pesquisador do ensino de História em seu país? Como isso favorece a formação de suas visões de mundo e de sociedade?

Sebastián Plá: Muy bien. Aunque soy un pez extraño en estas aguas. Soy el investigador en enseñanza de la historia con más publicaciones nacionales e internacionales, tengo bastantes alumnos y soy investigador de uno de los dos centros de investigación educativa más reconocidos de México. Pero al mismo tiempo casi nadie en México comparte mi visión - quizá cada vez más radical - política y cultural (y cada vez menos historiográfica) de la enseñanza de la historia, por lo que ahora trato de publicar más en el extranjero.

Lucia Valencia: La didáctica es un campo que se está abriendo paso en la investigación y en la academia universitaria. Ha necesitado de importantes procesos de legitimación al interior de los Departamentos donde la investigación ha sido por definición la investigación histórica.

Creo que hoy está en un momento de posicionamiento sin precedentes y que las expectativas sólo son auspiciosas. Este campo de investigación se ha ido consolidando como un referente con perspectivas, intereses y visiones propias del mundo y la sociedad y que cobra relevancia porque se relaciona directamente con la influencia que estas pueden llegar a tener en el mundo escolar, 
que sigue siendo el principal campo de socialización de la humanidad en el mundo moderno.

Martha Cecília: En este momento hay un buen ambiente en la comunidad interesada en la didáctica de la historia y de las ciencias sociales, hay avances y programas de formación avanzada, que empiezan a mostrar producción académica, que esperamos que impacte los currículos y las aulas de clase.

Las asociaciones y redes, como la de Didáctica de las Ciencias Sociales, nos han permitido empezar a escucharnos, a compartir la producción académica, los problemas e intereses, que nos ayuden a hacer de este campo del conocimiento, una posibilidad para la transformación de la enseñanza de la historia y las ciencias sociales en general.

Miguel Ángel: En mi país sucede “casi” lo mismo que en el resto del mundo iberoamericano, hay propuestas de investigación y formación docente enmarcadas en la tecnología educativa y muchas políticas públicas también se escudan detrás de esta perspectiva, podría afirmar que las corrientes críticas no son mayoritarias, pero en el marco de la Asociación de Profesores/as de Enseñanza de la Historia de Universidades Nacionales (APEHUN) hemos construido un núcleo de investigaciones educativas cualitativas y situadas en contexto que nos permite dar cuenta de la enorme diversidad de perspectivas para pensar y accionar las enseñanza de la historia.

En estos momentos en el seno de la Asociación estamos trabajando para articular acciones de investigaciones comparadas y comparativas entre las diversas regiones de la extensa Argentina, así que en ese sentido tenemos un recorrido en común y un amplio horizonte de expectativas porque nuestra región necesita de ciudadanas y ciudadanos críticos y comprometidos en valores que indican mejor distribución de lo que producimos y mejor acceso a bienes materiales y culturales y sabemos que una buena educación histórica es central.

Una línea muy potente, en este sentido, establece la relación entre enseñanza de la historia y construcción de ciudadanías, apostamos a que desde la primera infancia se construyan prácticas de ciudadanización en infantes, niñas y niños.

Si observamos con atención las producciones y preocupaciones que ocupan a diversos equipos de investigaciones en nuestro país o en Colombia (los que integran la RCGIDCS - Red Colombiana de Grupos de Investigación en Didáctica de las Ciencias Sociales), o en Brasil, o en Chile; como así también en otros países 
como en España la AUPDCS (Asociación Universitaria de Profesorado de Didáctica de las Ciencias Sociales) y GREDICS en Barcelona (Grupo de Recerca en Didáctica de les Ciencies Socials), por nombrar solo algunos, estaríamos en condiciones de sostener y destacar la magnitud que ha cobrado la actividad y el importante desarrollo de las investigaciones en las últimas décadas. Esto me lleva, a pensar y valorar como positiva la producción, aunque no suficiente.

Sonia e Júnia: Tendo em vista as suas utopias, no que você acredita como uma aula de história "ideal”?

Sebastian Plá: Con las condiciones de las aulas mexicanas, primero pediría que se satisficieran las necesidades laborales de los docentes, se mejoraran las instalaciones (según el último censo cerca de 100,000 escuelas tienen algún problema de infraestructura: sin salones de clase, sin agua, sin electricidad, sin baños o sin drenaje) y desaparecieran las condiciones de marginación de los alumnos y sus familias, desde la alimentación hasta el poder adquisitivo (48 o más millones de pobres, de 107 millones de habitantes). Como eso no va a suceder con la nueva reforma educativa (2013), mi aula ideal de historia - más allá de lo laboral, social y material - sería una clase en la que la historia sirviera fundamentalmente para hacer consciente a los individuos de los procesos de dominación en los que nos encuentramos. Me refiero a las condiciones de clase social, pero sobre todo a la violencia simbólica y al régimen epistemológico que se nos impone. La historia y la nueva enseñanza de la historia, fundamentada en una visión blanca, sajona y occidental, universalizante, evolutiva y teleológica que se oculta detrás de las competencias y ciertas ideas del método del historiador y del pensar históricamente, puede que sirva para formar ciudadano en Canadá o Londres, pero no en el Sur. Por tanto, sería un aula donde se permitieron diferentes regímenes de historicidad y diferentes formas culturales de articular el sentido histórico. No me refiero a un pluralismo light donde las distintas interpretaciones de la historia son toleradas dentro de una forma única y evolutiva de pensar la historia, sino un aula en que las relaciones entre historias se den de manera agonística y en ocasiones, pues es inevitable, antagónicas. Una enseñanza situada, una enseñanza de la historia desde y para el Sur. Como hacer eso, todavía no tengo la repuesta, pero la estoy buscando y el camino es por la justicia curricular. 
Lucia Valencia: Es una pregunta muy difícil de responder, pero me parece que un aula de Historia ideal debe dar cuenta en su composición de la diversidad que caracteriza a las sociedades en que se inserta. Un aula de Historia ideal debe estar estrechamente vinculada con la sociedad y con la realidad de la que forma parte, tanto en sus finalidades como en sus experiencias de aprendizaje.

Un aula de Historia ideal debe contribuir a comprender esa realidad, cómo se ha conformado historicamente, las decisiones y acciones que se llevaron a cabo para permitirla y las decisiones y acciones que en el presente están trazando nuestro futuro.

Martha Cecília: Para mí no hay aula ideal, el aula de historia y de ciencias sociales debe estar permeada por problemas socialmente relevantes o por cuestiones socialmente vivas, en las que estudiantes y docentes dialoguen, indaguen, debatan, argumenten y contra argumenten, con el objeto de construir otras miradas de la historia y del conocimiento social, más críticas, complejas y reales, que incluyan las distintas visiones y versiones de los hechos y fenómenos sociales, que contribuyan a la formación del pensamiento social y a la construcción de ciudadanos críticos, participativos y comprometidos con la construcción de sus comunidades.

Miguel Ángel: Bueno, la utopía ya no está de moda en algunos ámbitos intelectuales, pero nuestras sociedades modernas por más desencantadas que estén no dejan de producir sus propios futuros y en los que la dimensión de lo político se convierte en un terreno propicio para la construcción de representaciones imaginarias colectivas, en términos de lo que plantea Bronislaw Baczo, y en ese sueño de una sociedad distinta la historia y su enseñanza tienen mucho por hacer. Me parece que después de los acontecimientos traumáticos del siglo pasado y del que estamos viviendo, necesitamos una educación que vuelva a pensar la estrecha relación entre historia y política y que el aula de historia sea un espacio de socialización política para las y los adolescentes y jóvenes.

Otra temática que cobra importancia en el mundo en que vivimos es la relación historia memoria e identidad, en un mundo de identidades múltiples es necesario advertir la diversidad de abordajes no sólo teóricos sino también prácticos, vivimos en un mundo que dice que reconoce la diversidad pero que rara vez la respeta, vivimos en un mundo muy xenófobo y prueba de ello es el tratamiento que se le da a las migraciones y a las y los migrantes. 
Una temática muy estudiada en vuestro país es la relación entre historia, enseñanza y conciencia histórica y es a partir de ella que podemos construir futuros, que podemos enseñar los múltiples futuros posibles.

En un trabajo reciente, realizado en la zona en la que vivo (el Alto Valle del Río Negro y Neuquén en la Nord Patagonia), Graciela Funes articula la enseñanza de la historia a la construcción de una ciudadanía crítica. Sostiene que la ciudad de Neuquén (sede de la UNCo) tiene una rica trayectoria en enseñanza de historias recientes, derechos humanos y de pensar a la historia y su enseñanza como un lugar para la transmisión de cultura, de construcción de futuro, de comprensión de la realidad social, de socialización política y estos son lugares siempre de creatividad utópica ligada a la historia que se extienden y se intensifican porque siempre es posible acceder a más derechos, ser más críticos, más creativos, más democráticos, más solidarios. Es aquí donde encuentro aires de lo posible.

Una síntesis del aula que aspiro a construir, parafraseando al maestro Freire, es aquella que este abierta y posibilite la discusión valiente de su problemática, nos invite al dialogo con el otro y se disponga a un análisis crítico de sus descubrimiento. Así pensada, se trata de construir un aula que promueva más preguntas en vez de ofrecer respuestas acabadas.

Por otra parte y más allá de los programas de formación de las profesoras y de los profesores en historia y de sus límites, podemos pensar estrategias que nos ayuden a cambiar la enseñanza y la formación del profesorado de historia focalizando en una historia para la ciudadanía, una historia que explique problemas sociales y brinde elementos para una participación activa en la toma de decisiones.

Redefinir, entonces, la formación inicial y continuada presupone partir de la consideración de estos aspectos. No alcanza sólo con los saberes de las disciplinas. Debemos pensar que formar para la enseñanza de un objeto inacabado y en permanente construcción, como lo es la historia, exige educar en y para una racionalidad que no ponga límites, así como fortalecer la capacidad crítica que traspase la perspectiva cosificada del saber-información.

Artigo recebido em 24 de setembro de 2014. Aprovado em 23 de outubro de 2014. 